\title{
Morroniside promotes bone marrow mesenchymal stem cell proliferation in rats
}

\author{
NAIWU HU ${ }^{1}$, SHUJUN REN $^{2}$, WEI LI $^{2}$, TAO ZHANG ${ }^{1}$ and CHENGBIN ZHAO ${ }^{1}$ \\ ${ }^{1}$ Department of Orthopedic Surgery, The Fourth Hospital of Harbin Medical University, Harbin, \\ Heilongjiang 150001; ${ }^{2}$ Department of Orthopedics and Traumatology, The First Affiliated Hospital \\ of Heilongjiang University of Chinese Medicine, Harbin, Heilongjiang 150040, P.R. China
}

Received November 7, 2012; Accepted February 15, 2013

DOI: $10.3892 / \mathrm{mmr} .2013 .1399$

\begin{abstract}
The aim of this study was to explore the mechanism underlying the promotive effect of morroniside on rat mesenchymal stem cell (RMSC) proliferation and to provide an experimental basis for the development of potential new drugs. RMSCs were obtained from the bone marrow of Sprague-Dawley rats aged 3-4 months. The proliferation of primary and subcultured RMSCs in the high-, mediumand low-concentration morroniside intervention and blank control groups was observed using light microscopy. Cell proliferation and survival conditions were detected using methyl thiazolyl tetrazolium (MTT) colorimetric tests. Light microscopy and the MTT assay revealed that RMSC adherence time in the morroniside groups was shorter compared with that of the control group. Twelve hours after the media of primary RMSCs were changed, the number of adherent cells in the morroniside groups increased and an elongated cell morphology was observed. The cells at the fourth passage in the morroniside groups fused completely $12-16 \mathrm{~h}$ after inoculation and then rapidly entered into the logarithmic phase. The primary RMSCs of the morroniside intervention groups grew into typical bone marrow mesenchymal stem cell (BMSC) colonies after 4 days of morroniside treatment and their fusion rate had reached $80 \%$ after 9-11 days. By contrast, the cell fusion rate of the control group only reached $75-80 \%$ after 14 days of morroniside treatment. Morroniside exhibited a similar promotive effect on proliferation in primary and subcultured RMSCs. Morroniside may promote RMSC proliferation through secreted factors, cell-to-cell interactions and/ or the interactions between cellular adhesion molecules and extracellular matrices (ECMs). However, the specific mechanism underlying this effect remains to be fully elucidated.
\end{abstract}

Correspondence to: Professor Chengbin Zhao, Department of Orthopedic Surgery, The Fourth Hospital of Harbin Medical University, No. 37 Yiyuan Street, Harbin, Heilongjiang 150001, P.R. China

E-mail: naiwuhuchina@yeah.net

Key words: morroniside, rat, bone marrow mesenchymal stem cell, proliferation

\section{Introduction}

Mesenchymal stem cells (MSCs) are multipotent stromal cells that differentiate into histiocytes of the ectoderm, mesoderm and endoderm. MSCs are able to directly differentiate into osteoblasts and chondroblasts in vivo and in vitro. Since they are the source of osteogenitor cells, MSCs constitute the cytological basis of osseous tissue regeneration. Due to their multi-directional differentiation potential (1), MSCs have become the most important seed cells in the field of tissue and cell engineering and their potential application value has attracted increasing amounts of attention. As a result, the basic and clinical investigation of MSCs has gradually become the focus of several studies on stem cells worldwide.

Trauma, infection and various bone diseases cause bone non-union and deletion. When bone non-union and deletion occur, the commonly adopted treatment method is bone grafting; however, conventional bone grafting has a number of limitations. Although autogenous bone grafting has a good curative effect and does not cause immunogenicity, it is restricted by the limited amount of bone it is able to harvest and extra injury it may inflict at the bone supply area. Although xenogenous bone grafting is not restricted by the same limitations as autogenous bone grafting, it has the potential to induce a number of problems, including immune reactions and disease transmission. As tissue engineering develops, in vitro constructed artificial bones are expected to completely reconstruct injured bone tissues without leading to severe complications $(2,3)$. At present, bone tissue engineering research mainly includes the in vitro culture of seed cells, research and development of matrical materials for cell implantation and investigation of the regulatory effects of various factors in tissue cultures (4). Bone marrow mesenchymal stem cells (BMSCs) may be obtained by adherent culture and isolation in vitro and have a rapid proliferation rate. Due to their multiple differentiation potentials, BMSCs may differentiate into various tissue cells, including osteoblasts, chondrocytes, tendon and fat cells, fibroblasts and neural satellite cells. Furthermore, BMSCs have a strong self-duplicating ability. As a result of these characteristics, BMSCs have become an important type of seed cell for application in tissue engineering (5-7). However, various problems with regard to the application of BMSCs as seed cells remain to be clarified 
in bone tissue engineering, including the effective collection of BMSCs from bone marrow in unit volume, accurate and effective induction of BMSC differentiation into osteoblasts without causing damage, the ability to guarantee the differentiation of implanted BMSCs into osteoblasts and exertion of their bone formation function (8).

The proliferation and differentiation of stem cells are strictly regulated. Due to the existence of these regulatory mechanisms, the group stability of stem cells shown in various tissues is termed autostability. However, stem cells proliferate and differentiate under various physiological or pathological conditions, indicating the existence of strict regulation (9). Currently, the regulatory mechanisms of stem cells are considered to be determined by the microenvironments of organ tissues, known as stem cell niches (10). A niche is a dynamically balanced local environment which is composed of stem cells, surrounding cells and factors associated with the regulation of proliferation and differentiation (11). Excreted factors, cell-to-cell interactions and the interaction between integrin and the extracellular matrix (ECM) are crucial in maintaining the balance of the niche. Excreted factors in a niche are excreted by stem cells in the niche or other cells and tissue cells surrounding the niche. They are important in the proliferation and differentiation of stem cells (12). In different stem cell microenvironments and physiological or development states, the types and levels of excreted factors may vary; however, these variations always meet the requirements for development or physiological states. With the exception of free excreted factors, signaling molecules that regulate stem cell fate exert their effect on stem cells via cell-to-cell interactions in the niche. These interactions are normally mediated by integral membrane proteins. Integral proteins are a type of cell membrane-bound protein; although they are known to belong to the group of transmembrane proteins, they are different from peripheral and anchoring proteins $(13,14)$. All the integral transmembrane proteins take the form of amphipathic molecules (15). Numerous mediator cell and cellular adhesion molecules are subject to integral membrane proteins and are important in stem cell proliferation. Integrin is a type of cellular adhesion molecule that mediates cell-to-cell and cell-to-ECM interactions and these functions stem from $\mathrm{Ca}^{2+}$. Integrin is expressed in the majority of animal and plant cells and its role is to correctly place stem cells in tissues to avoid their differentiation or death as a result of being isolated from their living microenvironment $(14,16,17)$.

Morroniside is the primary active component of dogwoods and elderberries. It has wide bioactivity and promotes osteoblast proliferation in vitro $(18,19)$. Morroniside belongs to a group of iridoid glucosides, whose compounds have a wide bioactivity, including antiviral, antibiotic, antitumoral, antioxidant, hepatoprotective, choleretic, spasmolytic, analgesic, immune strengthening and sugar/lipid reducing properties. Additionally, they benefit the nervous, cardiovascular and digestive systems $(20,21)$.

In the present study, the effect of morroniside on BMSC proliferation was investigated. The aim of the present study was to explore methods for the amplification of BMSCs, thereby providing more suitable seed cells for bone tissue engineering. Furthermore, we aimed to provide an experimental basis for the mechanism of action of morroniside and for the development of potential new drugs.

\section{Materials and methods}

Animals. Healthy 3 to 4-month-old Sprague-Dawley rats of either gender (22) were provided by the Animal Experimental Center of the Harbin Medical University (Harbin, China).

This study was performed in strict accordance with the recommendations in the Guide for the Care and Use of Laboratory Animals of the National Institutes of Health. The animal use protocol was reviewed and approved by the Institutional Animal Care and Use Committee (IACUC) of the Fourth Affiliated Hospital of Harbin Medical University.

Morroniside extraction and intervention liquid preparation. Morroniside (348 $\mathrm{g}$ in total) was extracted and the obtained product was compared with the reference standard supplied by the Tianjin Kuiqing Trading Company (Tianjin, China; HPLC>98; 20 mg/package; purity, 95\%). Sufficient resolutions were achieved by adding morroniside $(10 \mathrm{mg})$ to a small amount of dimethyl sulfoxide (DMSO). This was prepared into 10,100 and $800 \mu \mathrm{g} / \mathrm{ml}$ working solutions with phosphate-buffered saline (PBS). The solutions were filtrated with a pinhole filter $(0.2 \mu \mathrm{m})$, sterilized, subpackaged and then stored at $4^{\circ} \mathrm{C}$.

Rat BMSC (RMSC) isolation and treatment of primary RMSCs with morroniside. The rats (average weight, 200 $\pm 10 \mathrm{~g}$ ) were sacrificed using cervical dislocation and soaked in $70 \%$ ethanol for 5-10 min. The tibia and femur were isolated on a clean bench and the soft tissues attached to the bones were removed. The metaphysis was cut open using sterilized eye scissors and the marrow cavity was flushed with serum-free Dulbecco's modified Eagle's medium (DMEM) containing low amounts of carbohydrate for cell suspension preparation. Percoll separating medium $(1.073 \mathrm{~g} / \mathrm{ml})$ in the same volume was prepared. The cell suspension was slowly added to the medium (1:1 ratio) along the wall of the centrifuge tube and then centrifuged at $716 \mathrm{x}$ g for $20 \mathrm{~min}$ at room temperature. The cloud-like cell layer between the liquid levels was carefully absorbed, while the white liquid of the lower level was avoided. The obtained product was blown softly with PBS (0.01 M) for the cell suspension. The suspension was centrifuged at $179 \mathrm{x} g$ for $5 \mathrm{~min}$ at room temperature and the supernatant was discarded. The remaining product was washed again with PBS $(0.01 \mathrm{M})$, centrifuged at $179 \mathrm{x} \mathrm{g}$ for $5 \mathrm{~min}$ at room temperature and the supernatant was removed. DMEM containing $10 \%$ PBS was used for the cell suspension preparation. The cells were counted using a microscope and inoculated in 6-well plates at a concentration of $2 \times 10^{6} / \mathrm{ml}$. The cells were divided into four groups; the low-, medium- and high-morroniside and control groups. Six wells were set for each group. The cells were cultured in a $5 \% \mathrm{CO}_{2}$ incubator in $100 \%$ humidity at $37^{\circ} \mathrm{C}$. The culture medium was first changed after $48 \mathrm{~h}$. Morroniside fluid was added to maintain the drug concentrations of the morroniside intervention groups at 2 (low), 20 (medium) and $200 \mu \mathrm{g} / \mathrm{ml}$ (high). During the period of intervention culture, the volume ratio between the drug and the culture liquid $(<1 / 4)$ was maintained. The culture medium for each group was changed 
every three days. The primary cells were observed under an inverted phase contrast microscope.

Treatment of subcultured RMSCs with morroniside. RMSCs at the fourth passage were trypsinized for a monoplast suspension. The cells were divided into four groups; the low-, medium- and high-morroniside and control groups. The cells were inoculated in a 24-well plate at a concentration of $2 \times 10^{3} / \mathrm{ml}$ and were adhered to the walls after $36 \mathrm{~h}$. The culture solution in each well was replaced with serum-free culture medium. Cell cycle synchronization occurred after $24 \mathrm{~h}$ and morroniside was added. The drug concentrations of the low-, medium- and high-morroniside intervention groups were maintained at 2, 20 and $200 \mu \mathrm{g} / \mathrm{ml}$, respectively. During the intervention culture, the volume ratio between the drug and the culture liquid $(<1 / 4)$ was maintained. The cells were observed under a microscope.

Methyl thiazolyl tetrazolium (MTT) assay. An MTT assay, also known as MTT colorimetry, is a method used for the detection of cell survival and growth. This detection is possible due to the presence of succinate dehydrogenase in the mitochondrion of living cells, which reduces ectogenic MTT into formazan, a water-insoluble blue-purple crystal, and this is deposited among the cells. The amount of MMT crystals are directly proportional to the number of cells present.

RMSCs at the fifth passage were trypsinized for a monoplast suspension. The cells were divided into four groups; the low-, medium- and high-morroniside and control groups. The cells were inoculated in a 96-well plate at a concentration of $2 \times 10^{3} / \mathrm{ml}$ and were adhered to the walls after $36 \mathrm{~h}$. The culture solution in each well was replaced with serum-free culture medium. The cell cycles were synchronized after $24 \mathrm{~h}$. Morroniside was added and the drug concentrations of the low-, medium- and high- morroniside intervention groups were maintained at 2, 20 and $200 \mu \mathrm{g} / \mathrm{ml}$, respectively. During the intervention period, the volume ratio between the drug and the culture solution was maintained at $<1 / 4$. Six wells from each group were digested every two days, following the addition of the drug. The culture solution was discarded and the cells were washed three times with D-Hanks. The cells were cultured with $90 \mu \mathrm{l}$ of serum-free DMEM medium and $5 \mathrm{mg} / \mathrm{ml} \mathrm{MTT}(10 \mu \mathrm{l} /$ well $)$ in a $5 \% \mathrm{CO}_{2}$ incubator at $37^{\circ} \mathrm{C}$ for $4 \mathrm{~h}$. The supernatant was discarded and DMSO (150 $\mu \mathrm{l} /$ well) was added and oscillated for $10 \mathrm{~min}$. Optical density (OD) was read at wavelengths of 570 (measured wavelength) and $655 \mathrm{~nm}$ (reference wavelength) and the wells which contained no cells were evaluated as zero adjustment wells. The results were recorded and growth curves were drawn, taking time as the horizontal axis and OD as the longitudinal axis.

Statistical analysis. Data were presented as the mean \pm standard error of the mean (SEM) or as percentages $(100 \%)$. One-way ANOVA was performed using SPSS13.0 software (for Windows).

\section{Results}

Effect of morroniside on primary RMSC proliferation. At $24 \mathrm{~h}$ after inoculation, a number of cells had adhered to the
A

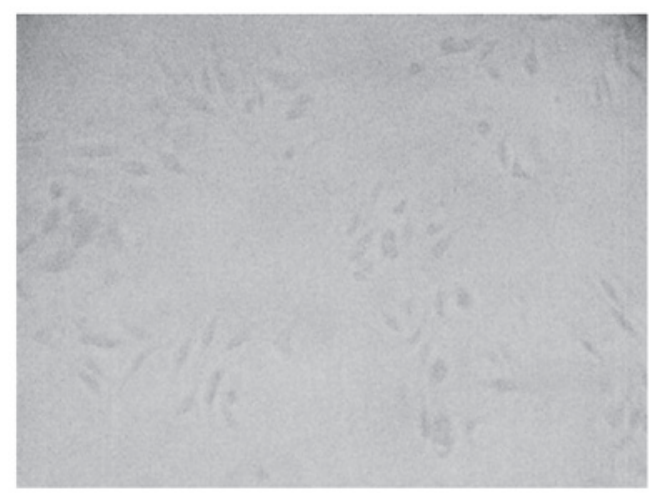

B

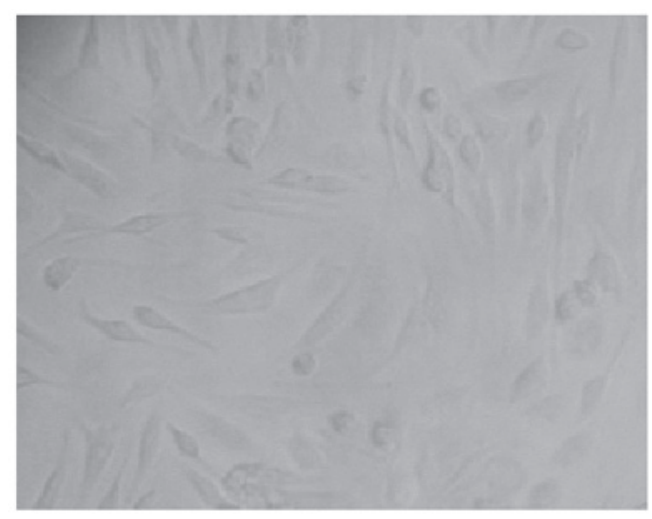

C

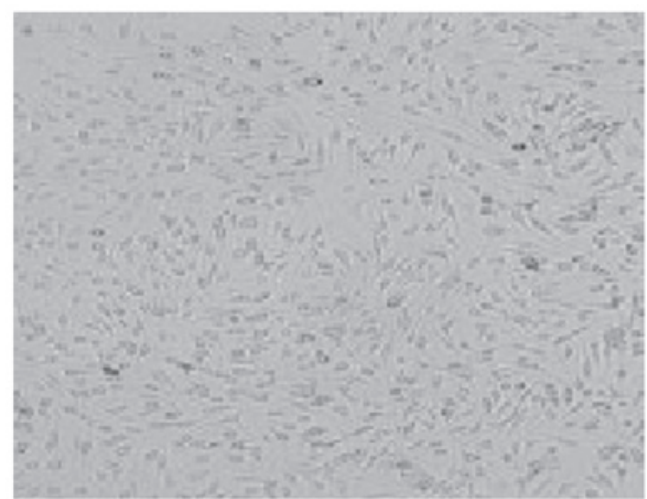

Figure 1. (A) Rat mesenchymal stem cells (RMSCs) after 24 h, (B) 6 days and (C) 11 days of morroniside treatment.

wall and were characterized by a spindle-or star-like diffused distribution. After $48 \mathrm{~h}$, the media were changed and the non-adherent cells were removed. The remaining cells were carefully washed with PBS. Morroniside liquid was added to maintain the final drug concentrations of the low-, mediumand high-concentration intervention groups at 2, 20 and $200 \mu \mathrm{g} / \mathrm{ml}$, respectively. Twelve hours after the medium was changed, the amount of adherent cells increased in all the intervention groups and a number of cells began to adopt an elongated morphology. After $24 \mathrm{~h}$, the number of adherent cells was further increased. There were marked cellular processes, with large and oblate nuclei. The phenomenon of cell division was occasionally observed. After 4 days of morroniside treatment, typical BMSC colonies emerged and these varied in size. Subsequently, the number of the colonies gradually increased and adherent cells in radial growth were observed around the centers of the colonies. These cells had 
Table I. OD values of RMSC in different groups using an MTT assay (mean \pm SEM).

Duration of morroniside treatment

\begin{tabular}{|c|c|c|c|c|c|c|}
\hline \multirow{2}{*}{$\begin{array}{l}\text { Morroniside } \\
\text { treatment }(\mu \mathrm{g} / \mathrm{ml})\end{array}$} & & & & & & \\
\hline & 1 day & 3 days & 5 days & 7 days & 9 days & 11 days \\
\hline 0 (control) & $0.1121 \pm 0.0032$ & $0.1514 \pm 0.0329$ & $0.2519 \pm 0.0631$ & $0.3564 \pm 0.0501$ & $0.5850 \pm 0.0209$ & $0.6412 \pm 0.0095$ \\
\hline 2 (low) & $0.0845 \pm 0.0041$ & $0.2677 \pm 0.0541$ & $0.3995 \pm 0.0848$ & $0.6236 \pm 0.0245$ & $0.6516 \pm 0.0351$ & $0.6919 \pm 0.0211$ \\
\hline 20 (medium) & $0.0942 \pm 0.0102$ & $0.2694 \pm 0.0706$ & $0.3654 \pm 0.0106$ & $0.5851 \pm 0.0304$ & $0.6414 \pm 0.0106$ & $0.6860 \pm 0.0309$ \\
\hline 200 (high) & $0.0945 \pm 0.0248$ & $0.2802 \pm 0.0354$ & $0.3632 \pm 0.0059$ & $0.5704 \pm 0.0741$ & $0.6553 \pm 0.0215$ & $0.6720 \pm 0.0291$ \\
\hline
\end{tabular}

RMSC, rat mesenchymal stem cell; OD, optical density; SEM, standard error of the mean.

A

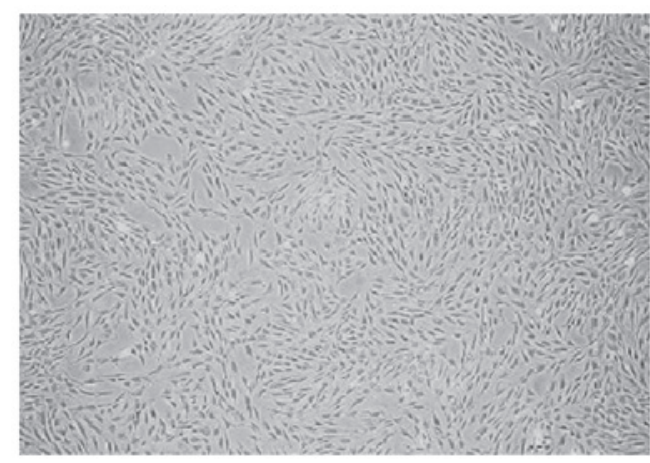

B

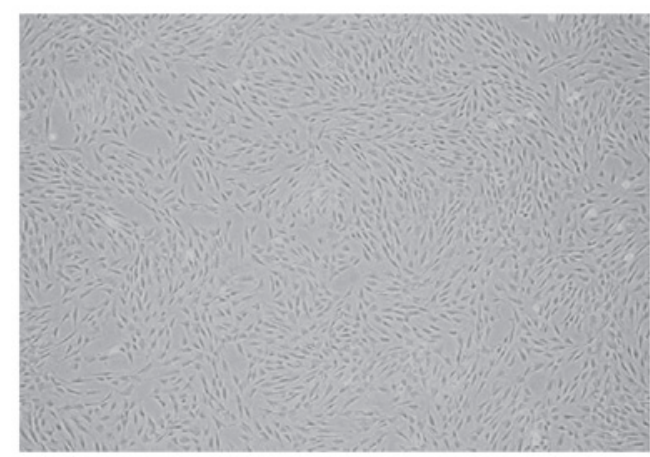

Figure 2. (A) Mesenchymal stem cells (MSCs) at the fourth passage after (A) 7 and (B) 11 days of morroniside treatment in the medium concentration group.

large bodies with a uniform configuration (the majority were spindle-shaped) and were arranged compactly. Thereafter, cells proliferated rapidly. At days 6-7, cells in the colonies converged and the occurrence of cell division was reduced. After 9-11 days of morroniside treatment, compact cells were located at the centers of the colonies and a cell fusion rate of $80 \%$ was achieved. No significant differences were observed among the morroniside intervention groups. The cells gradually fused into a single layer and were arranged in order along the long axis of the cell bodies, exhibiting an eddy-like form. Compared with the morroniside intervention groups, cells in the control group proliferated slowly; the colonies emerged after 9-11 days and the cell fusion rate had only reached $75-80 \%$ on day 14 (Fig. 1).

Effect of morroniside on subcultured RMSC proliferation. Cells at the fourth passage in the morroniside intervention

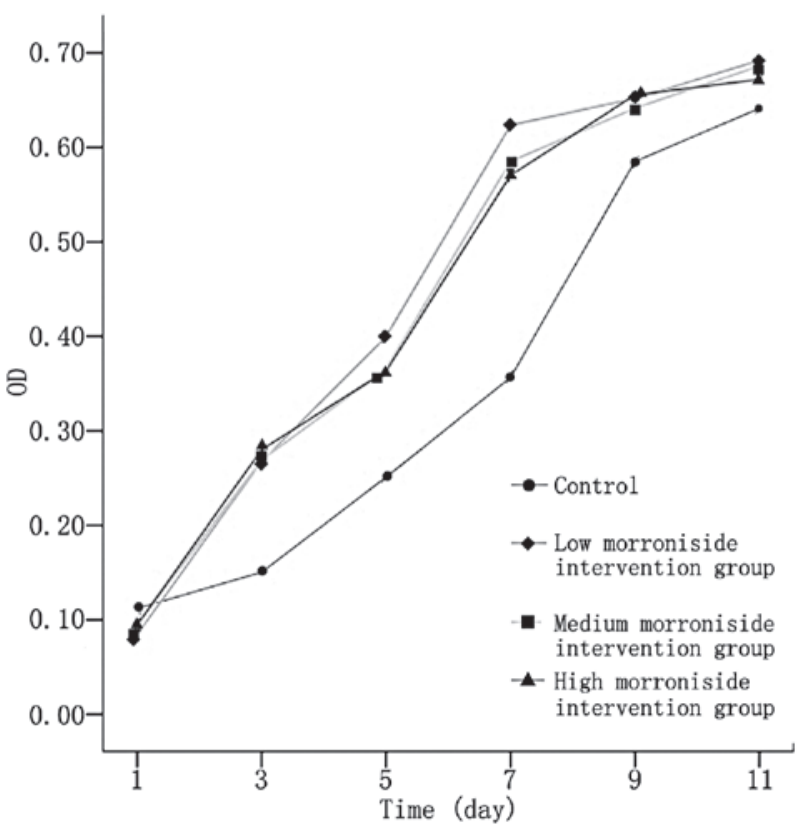

Figure 3. Rat mesenchymal stem cell (RMSC) growth curves for the four groups. The proliferative activity was detected using an MTT assay.

groups were not significantly different compared with those in the control group when examined under a microscope. The cells were spindle- or star-shaped with large nuclei and decreased plasma and the majority of cells had one or two nucleoli and were arranged in a radial or eddy-like manner. Twelve to $16 \mathrm{~h}$ after cell inoculation, the fourth passage cells were completely adhered to the walls and had returned to the spindle- or star-like shape; they entered a platform phase after 7-9 days of morroniside treatment. The cells in the control group adhered to the walls after $\sim 24 \mathrm{~h}$ and entered a platform phase after 7-8 days. Cells in different groups and at different passages were not significantly different; their morphology was similar, they had a uniform configuration and no cells grew in suspension (Fig. 2).

MTT assay. As shown in Fig. 3 and Table I, cells in the morroniside intervention groups entered into logarithmic growth immediately after the media were changed for subculture, which is a markedly shorter time for RMSC suspension and adherence in these groups. By contrast, the cells in the control group only entered a logarithmic growth phase at 
day 3. These findings are consistent with the considerations in the first section of the present study. The $2-\mu \mathrm{g} / \mathrm{ml}$ morroniside group entered a platform phase after 7 days and the $20-$ and $200-\mu \mathrm{g} / \mathrm{ml}$ groups entered after 7-9 days, whereas the control group only entered the platform phase after 9-11 days. Furthermore, one-way ANOVA analysis showed significant differences between the morroniside intervention groups and the control group between days 3 and $7(\mathrm{P}<0.05)$, while one-way ANOVA multiple comparisons did not identify significant differences among the morroniside intervention groups $(\mathrm{P}>0.05)$. However, no significant differences were identified in any of the groups after 9-11 days $(\mathrm{P}>0.05)$.

\section{Discussion}

The present study demonstrates that morroniside exerts an effect on the proliferation of primary and subcultured RMSCs. The treatment of the primary RMSCs with morroniside caused the amounts of adherent cells in all morroniside groups to increase $12 \mathrm{~h}$ after the media was changed and the cells were characterized by an elongated morphology. After $24 \mathrm{~h}$ of morroniside treatment, a large number of adherent cells appeared. These cells had large and oblate nuclei, with marked cellular processes and occasional cell division. After 4 days, typical BMSC colonies of varying sizes were observed in these groups. Subsequently, the number of BMSC colonies gradually increased in the morroniside groups and adherent cells grew radially from the centers of the colonies. These were characterized by a uniform morphology (the majority were spindle-shaped) with large bodies and in a compact arrangement. Thereafter, the cells proliferated rapidly; the cells in the colonies converged and the occurrence of cell division was reduced after 6-7 days. After 9-11 days, cells grew compactly around the centers of the colonies and a cell fusion rate of $80 \%$ was achieved. Different morroniside groups were not significantly different; the cells gradually fused into a single layer and were arranged in an eddy-like form, in an orderly manner along the direction of the long axis of their bodies. By contrast, cells in the control group proliferated slowly. When compared with the cells in the morroniside groups in the corresponding period, colonies were only formed in the control group after 9-11 days and reached a fusion rate of $75-80 \%$ after 14 days. The investigation of the effect of morroniside on subcultured RMSC proliferation demonstrated that cells at the fourth passafe were completely adhered and returned to a spindle or star shape 12-16 h following inoculation. They entered into a platform phase after 7-9 days of treatment. By contrast, the cells in the control group adhered after $24 \mathrm{~h}$, and they entered a platform phase after 9-11 days. The cells of different groups and passages presented with a uniform configuration, exhibited no significant differences in morphology and no cells grew in suspension.

The above findings show that morroniside has a similar active promotive effect on proliferation in primary and subcultured RMSCs, as demonstrated by the following observations. i) Cells adhered early. Compared with the control group, increased amounts of cells in the intervention groups were adhered $12 \mathrm{~h}$ after the media were changed. The cells in the intervention groups were characterized by an elongated morphology. Furthermore, cells at the fourth passage in the intervention groups completely adhered to the walls within $12-16 \mathrm{~h}$ following inoculation; ii) the cells rapidly entered logarithmic growth. Typical BMSC colonies in the intervention groups emerged after 4 days and the cells reached a fusion rate of $80 \%$ after 9-11 days of treatment. By contrast, the cells in the control group fused after 14 days and the fusion rate was only 75-80\%. These results are consistent with our MTT assay results.

Table I and Fig. 3 show that RMSCs in different intervention groups grew rapidly following subculture, although they exhibited varying degrees of time delay (within 1-2 days). Subsequently, the cells in these groups entered logarithmic growth. However, the cells in the intervention groups entered into a logarithmic growth phase earlier compared with the cells in the control group. This phenomenon may be correlated with early cell adherence in these groups. The cells in the intervention groups entered a growth platform phase after 7-9 days of treatment, which was earlier compared with those in the control group. Furthermore, the OD values of different intervention groups were not significantly different between days 7 and 11 after treament. This may due to the complete fusion of cells, resulting in their inhibition of each other upon contact.

MSC proliferation may be explained from the perspective of the conditions required for stem cell niches (23). Thus, it is reasonable to hypothesize that morroniside exerts its effect on the proliferation of primary and subcultured RMSCs through the following channels: i) morroniside may have a similar configuration to certain excreted factors in stem cell niches or it promotes the excretion of these factors through specific channels (such as the transcriptional level); ii) morroniside may strengthen the interactions between cells by binding with transmembrane proteins and promoting integral membrane protein to perform an anchoring role $(24,25)$; and iii) morroniside may work together with $\mathrm{Ca}^{2+}$ or strengthen the latter to mediate the interactions between cellular adhesion molecules and ECMs; this may be a crucial channel via which it carries out its role in MSC proliferation (26). However, these hypotheses are yet to be confirmed.

\section{References}

1. Bruder SP, Jaiswal N and Haynesworth SE: Growth kinetics, self-renewal, and the osteogenic potential of purified human mesenchymal stem cells during extensive subcultivation and following cryopreservation. J Cell Biochem 64: 278-294, 1997.

2. Long MW, Robinson JA, Ashcraft EA and Mann KG: Regulation of human bone marrow-derived osteoprogenitor cells by osteogenic growth factors. J Clin Invest 95: 881-887, 1995.

3. Taguchi Y, Yamamoto M, Yamate T, et al: Interleukin-6-type cytokines stimulate mesenchymal progenitor differentiation toward the osteoblastic lineage. Proc Assoc Am Physicians 110: 559-574, 1998.

4. Zhao K and Qin S: Bone tissue engineering research progress. J Liaoning Med Univ 1: 382-384, 2011 (In Chinese).

5. Carlo-Stella $\mathrm{C}$ and Gianni MA: Biology and clinical applications of marrow mesenchymal stem cells. Pathol Biol (Paris) 53: 162-164, 2005.

6. Kassem M: Mesenchymal stem cells: biological characteristics and potential clinical applications. Cloning Stem Cells 6: 369-374, 2004.

7. Okubo Y, Bessho K, Fujimura K, et al: Osteoinduction by recombinant human bone morphogenetic protein-2 at intramuscular, intermuscular, subcutaneous and intrafatty sites. Int J Oral Maxillofac Surg 29: 62-66, 2000.

8. Inao M, Mochida S and Matsui A, et al: Japanese herbal medicine Inchin-ko-to as a therapeutic drug for liver fibrosis. J Hepatol 41: 584-591, 2004. 
9. Hu Z, Zeng R, Guo W and Lin H: Induction and differentiation of bone marrow mesenchymal stem cells. J Clin Rehabil Tissue Eng Res 12: 8561-8566, 2008 (In Chinese).

10. Pontikoglou C, Deschaseaux F, Sensebé L and Papadaki HA Bone marrow mesenchymal stem cells: biological properties and their role in hematopoiesis and hematopoietic stem cell transplantation. Stem Cell Rev 7: 569-589, 2011.

11. Xia W, Xu R, Ye X, et al: Biological appraisal of human bone marrow mesenchymal stem cells during ex-vivo expansion. Zhongguo Shi Yan Xue Ye Xue Za Zhi 16: 639-644, 2008 (In Chinese).

12. Yoo HJ, Yoon SS, Park S, et al: Production and characterization of monoclonal antibodies to mesenchymal stem cells derived from human bone marrow. Hybndoma (Larchmt) 24: 92-97, 2005.

13. Weinreb M, Grosskopf A and Shir N: The anabolic effect of PGE2 in rat bone marrow cultures is mediated via the EP4 receptor subtype. Am J Physiol 276: E376-E383, 1999.

14. Kveiborg M, Flyvbjerg A, Eriksen EF and Kassem M: 1,25-Dihydroxyvitamin D3 stimulates the production of insulin-like growth-factor-binding proteins-2,-3 and -4 in human bone marrow stromal cells. Eur J Endocrinol 144: 549-557, 2001.

15. Tabin CJ: Retinoids, homeoboxes, and growth factors: toward molecular models for limb development. Cell 66: 199-217, 1991.

16. Gerhart TN, Kirker-Head CA, Kriz MJ, et al: Healing segmental femoral defects in sheep using recombinant human bone morphogenetic protein. Clin Orthop Relat Res 293: 317-326, 1993.

17. Locklin RM, Williamson MC, Beresford JN, Triffitt JT and Owen ME: In vitro effects of growth factors and dexamethasone on rat marrow stromal cells. Clin Orthop Relat Res 313: 27-35, 1995.

18. Pungitore CR, Ayub MJ, García M, et al: Iridoids as allelochemicals and DNA polymerase inhibitors. J Nat Prod 67: 357-361, 2004.
19. Li YM, Li H and Li CR: Advance on chemical compositions and pharmacology of Cornus officinalis. Acta Academiae Medicinae CPAF 6: 74-75, 2010.

20. Wang W, Sun F and An Y: Morroniside inhibits hydrogen peroxide-induced calcium overload and cytotoxicity in SH-SY5Y neuroblastoma cells. Chin J Rehabil Theory Pract 3: 115-117, 2009.

21. Zhang L, Li C and Zhao L: Effects of cornel iridoid glycoside on nervous function and neuron damage in focal cerebral ischemic rats. Chin J Rehabil Theory Pract 3: 110-113, 2007.

22. Javazon EH, Colter DC, Schwarz EJ and Prockop DJ: Rat marow stromal cells are more sensitive to plating density and expand more rapidly from single-cell-derived colonies than human marrow stromal cells. Stem Cells 19: 219-225, 2001.

23. McCabe LR, Banerjee C, Kundu R, et al: Developmental expression and activities of specific fos and jun proteins are functionally related to osteoblast maturation: role of Fra-2 and Jun D during differentiation. Endocrinology 137: 4398-4408, 1996.

24. Uludag H, D'Augusta D, Golden J, et al: Implantation of recombinant human bone morphogenetic proteins with biomaterial carriers: A correlation between protein pharmacokinetics and osteoinduction in the rat ectopic model. J Biomed Mater Res 50: 227-238, 2000.

25. Peng H, Wright V, Usas A, et al: Synergistic enhancement of bone formation and healing by stem cell-expressed VEGF and bone morphogenetic protein-4. J Clin Invest 110: 751-759, 2002.

26. Reddi AH: Bone morphogenetic proteins, bone marrow stromal cells, and mesenchymal stem cells. Maureen Owen revisited. Clin Orthop Relat Res 313: 115-119, 1995. 